\section{Cureus}

\title{
CyberKnife Ablation for Intramedullary Spinal Cord Arteriovenous Malformations (AVMs): A Promising New Therapeutic Approach
}

John R. Adler Jr. ${ }^{1}$, Gaurav Gupta ${ }^{2}$, Steven D. Chang ${ }^{2}$, Scott G. Soltys ${ }^{3}$, Iris C. Gibbs ${ }^{3}$, Gary K. Steinberg ${ }^{4}$, Michael B. Edwards ${ }^{2}$, Robert Dodd

1. Department of Radiation Oncology, Stanford University Medical Center, Stanford, CA, USA 2. Department of Neurosurgery, Stanford University School of Medicine 3. Department of Radiation Oncology, Stanford University School of Medicine 4. Stanford University School of Medicine 5.

$\square$ Corresponding author: John R. Adler Jr., jra@stanford.edu

Disclosures can be found in Additional Information at the end of the article

\section{Abstract}

Objective: Intramedullary spinal cord arteriovenous malformations (AVMs) are very rare lesions which are characteristically associated with recurrent hemorrhage and progressive ischemia. Although favorable location, size and vascular anatomy make it sometimes possible to manage this condition with embolization and/or microsurgical resection, there are no good treatment options for many, if not most, of these lesions. Given such poor prospects, the senior author began in 1997 investigating CyberKnife radiosurgical ablation as a tool for managing selected patients with intramedullary spinal cord AVMs. Although a previous paper in 2006 reported the preliminary experience from Stanford, our understanding has grown significantly as follow-up was extended and more patients were treated. Herein, we retrospectively analyze our longer term experience with a primary radiosurgical approach to spinal cord AVM.

Methods: Thirty patients (17 females: 13 males) with a median age of 33 years and intramedullary spinal cord AVMs (18 cervical, nine thoracic, and three conus medullaris) were treated with CyberKnife SRS between 1997 and 2008. A history of hemorrhage was present in $57 \%$ of cases, while in $50 \%$ of patients previous treatment included microsurgery $(6 / 29)$ or embolization (11/29). The mean AVM volume was $2.8 \mathrm{cc}(0.2-15 \mathrm{cc})$ and was treated to mean marginal dose of $20 \mathrm{~Gy}$ in one to four sessions (median 2). Based on our growing experience, the biologically effective dose (BED) of radiation was escalated gradually over the course of this study. Clinical and magnetic resonance imaging follow-up were carried out annually, and spinal angiography was repeated at three years.

Results: After a mean follow-up period of 63 months (median 54), the treated AVM was completely obliterated in eight cases (27\%) and significantly decreased in size in an additional

\section{Published 08/17/2010}

\section{(C) Copyright 2010}

Adler et al. This is an open access article distributed under the terms of the Creative Commons Attribution License CC-BY 3.0., which permits unrestricted use, distribution, and reproduction in any medium, provided the original author and source are credited.
11 cases (64\%) among the 22 angiographically studied patients with more than three years of follow-up. Pre-radiosurgery neurologic symptoms improved in more than $50 \%$ of cases, but worsened in three (10\%). There was one instance (3\%) of radiation-induced myelopathy occurring within one year of radiosurgery. Notably, after 158 years of collective post-SRS follow-up, no patient suffered a hemorrhage.

Conclusions: Although our understanding is still incomplete, CyberKnife radiosurgery results in total obliteration or shrinkage for most intramedullary spinal cord AVM, a phenomenon that may be accompanied by a marked decrease in the risk of bleeding. Symptom improvement is also common in most cases. More experience is required to determine the optimal radiosurgical 
dosing and consequent related efficacy. Given the significant therapeutic challenges inherent to spinal cord AVM patients, the rate of radiosurgical complications appears acceptable.

Categories: Radiation Oncology, Neurosurgery

Keywords: arteriovenous malformation, avm, cyberknife, hemorrhage, radiosurgery, spinal cord avm

\section{Introduction}

Intramedullary spinal cord arteriovenous SCAVMs are rare causes of spinal cord ischemia with a proclivity to affect young adults [1-5]. The estimated incidence of these lesions is only $0.02 / 100,000$ person-years while the prevalence is $0.08 / 100,000$ population [6-7]. Although the natural history is not well-characterized, historical studies and more recent case series suggest a significant risk of hemorrhage [3, 5, 8-9], often resulting in paralysis [1, 5]. Despite advances in microsurgery [10] and interventional neuroradiology [3], many of these lesions are still not amenable to such therapies because of their intraparenchymal spinal cord location and/or a common anterior spinal artery blood supply. Furthermore, the risk of complications from these established therapies, even in experienced hands, is considerable [3, 10, 11].

Although the feasibility of using stereotactic radiosurgery to ablate SCAVMs has previously been described [12-13], the extent of treatment benefits and the longer term risk of radiationinduced myelopathy both remain unknown. In this report, we describe our thirteen-year experience with CyberKnife radiosurgery for intramedullary SCAVMs focusing on its radiographic efficacy, alteration in the risk of hemorrhage, and change in clinical symptoms, while also assessing the risk of treatment-related complications.

\section{Materials And Methods}

\section{Patients}

Between August 1997 and November of 2009, 30 consecutive patients with angiographically visible intramedullary spinal cord arteriovenous malformations underwent CyberKnife stereotactic radiosurgery at Stanford University Medical Center under the auspices of the Institutional Review Board approved prospective study. Given the rarity of spinal cord AVMs, it is not surprising that many of our patients were referred nationally or internationally. Informed consent was obtained from each patient prior to beginning the radiosurgical process. Results of radiosurgery were retrospectively analyzed with radiographic response serving as the primary outcome measure. Secondary endpoints included rate of hemorrhage and improvement in clinical symptoms.

The selection criterion for radiosurgery involved only the presence of a symptomatic, angiographically-confirmed AVM within the spinal cord, which was felt to be associated with a high risk of spinal cord injury if resected or embolized. Almost all patients met this criterion by having a diffuse nidus encompassing nearly the entire cross sectional area of the spinal cord and/or a predominant blood supply via the anterior spinal artery. In 11 patients, previous endovascular embolization had been performed but residual nidus remained; the remnant being deemed too unsafe to occlude by means of embolization. Seven patients had residual AVM left after microsurgery for similar reasons. In cases of prior treatment (microsurgery or embolization), radiosurgery was administered only to regions of persistent pathologic blood flow on pre-radiosurgical angiograms.

\section{Radiosurgical technique}

A technical description of CyberKnife radiosurgery for spinal AVMs has previously been 
described [12]. In brief, all patients underwent repeat high-resolution MRI, CT angiography, and conventional selective spinal angiography to ascertain precise nidal volumes and establish their relationship with nearby skeletal landmarks. In the earliest years of this study, percutaneously-placed fiducial markers, utilized in the process of radiosurgical targeting, were surgically inserted prior to such imaging. Three-dimensional rotational angiography (3D DSA) was used routinely beginning in 2004. Qualitatively, such imaging provided superior identification of the AVM nidus separate from the adjacent draining veins [14]. Unsubtracted projection images from angiographic runs provided valuable clues to the spatial relationship between the AVM and adjacent skeletal landmarks. Of note, it was not technically possible, over the course of this series, to fuse time-dependent angiographic projection images (from 3D DSA) with the 3-D volumetric images. Actual target delineation took place on a combined data set (CT, MRI and the full 3D DSA volume) generated with semi-automated image fusion.

Treatment plans were generated with the CyberKnife inverse planning software seeking to optimize radiation dose conformality, while maximally sparing adjacent neural structures. Planning was an iterative process utilizing both qualitative assessments of conformality and a more quantitative analysis involving dose-volume histograms.

Following image-guided registration, radiosurgery was administered using standard CyberKnife procedures to awake, unanesthetized patients [12]. Partial immobilization was achieved with either an Aquaplast mask or alpha cradle mold depending on the spinal location being treated. Treatment times ranged between 40 to 90 minutes. After each radiosurgical session, patients were given $4 \mathrm{mg}$ of dexamethasone to prevent the exacerbation of adjacent spinal cord edema, and for some upper cervical lesions, anti-nausea medications were also administered.

\section{Radiosurgical dosage}

The selection of radiation dose throughout this series was the product of a complex calculus that drew upon an extensive radiosurgical experience with cerebral AVMs in critical deep brain regions [15], and later on, a growing understanding of the risks and benefits of using different doses to manage a range of intra- and extra-axial vascular and non-vascular spinal lesions [16]. At the initiation of this study, there was no prior experience with treating spinal lesions with radiosurgery. Primarily out of fear of inducing catastrophic spinal cord injury in oftentimes highly functional young patients, our initial dosing was deliberately quite conservative. To achieve this objective, a multi-session approach to radiosurgery was often employed, despite simultaneously recognizing that from standpoint of radiobiology, the use of fractionation to ablate AVMs may well be a self-defeating proposition $[12,17]$.

In comparing different fractionation regimens, conventional fractionated radiotherapy was used as a benchmark. From the linear quadratic model, a biologic equivalent dose (BED) can be estimated from the following formula: $\operatorname{BED}=\operatorname{nd}\left(1+d /\left(\alpha^{*} \beta\right)\right)$ [18]. Assuming an $\alpha$-to- $\beta$ ratio of $2 \mathrm{~Gy}$, multisession radiosurgical regimes were devised to gradually approach a BED comparable to a single session of $15 \mathrm{~Gy}$ which at the low end of dose used to treat some intracranial AVMs in critical locations; such a dose yields approximately a $70 \%$ rate of in-field obliteration for intracranial AVMs [19].

The final fractionation schedule (ranging over the course of the study between one to four sessions, with a median of two) was also customized to reflect AVM volume and spinal location. In the name of patient safety, dose selection repeatedly erred on the side of conservatism. Only gradually was the amount of radiation increased, largely by de-escalating the number of radiosurgical sessions. Prescription doses ranged between the 70 and $90 \%$ isodose contour lines and were defined at the margin of the AVM nidus. 


\section{Follow-up evaluations}

Clinical and radiographic evaluations were conducted at six months, one year, and annually thereafter. Patients were instructed that in the event they experienced any acute neurological deterioration they were to promptly contact us or their referring physician who in turn might communicate to us any pertinent information. In this regard, hemorrhage was defined as a clinically symptomatic event (sudden onset of pain, focal deficit, gait disturbance, genitourinary dysfunction, or combination of these) along with signs of fresh bleeding detected by means of CT or MRI. At six months and annually following radiosurgery, every patient underwent spinal MRI; at three years, spinal angiography was repeated. Angiographic obliteration was defined as the absence of abnormal vessels at the site of the original malformation, the disappearance or normalization of draining veins in and around the nidal region and a normal circulation time on angiography [20]. Neuroradiologists and neurosurgeons made independent determinations of AVM obliteration but with full knowledge of patients' histories.

AVM volume was estimated on two-dimensional angiograms by measuring nidal diameter in three orthogonal planes. This crude volume approximation utilized the formula for an idealized ellipsoid: $V=4 / 3\left(\pi r_{1} r_{2} r_{3}\right)$, where $r_{1}, r_{2}$, and $r_{3}$ denote the radius of the three cardinal planes. When three-dimensional angiography was performed, volumetric assessments were improved by being able to sum the cross-sectional areas of adjacent parallel slices. The validity of estimating AVM volume on projection angiography was semi-quantitatively corroborated in five patients who had both pre- and post 3D angiograms in addition to 2D studies.

If a SCAVM was not occluded on angiography, patients were asked to undergo repeated angiography 12 - 24 months later, or when an annual MRI suggested obliteration. In selected patients with residual AVM at five years post-radiosurgery, a second CyberKnife treatment was recommended. This was particularly pertinent for the earliest patients in this series to whom the most conservative radiation doses were prescribed. Of note, clinical follow-up continued even after total obliteration.

Information on all patients was prospectively entered into a computer database at the time of radiosugery and during subsequent clinic visits. The dates of diagnosis, radiosurgery, previous and additional hemorrhage, angiographic obliteration status, and last clinical follow-up were included in the analyses, along with data on the initial clinical presentation and treatment history. The observation period was divided into: the interval between symptom onset and radiosurgery, the interval between radiosurgery and angiographic obliteration, and the interval from angiographic obliteration to last clinical follow-up. The ascertainment of a hemorrhagic presentation prior to radiosurgery was determined by a history of acute onset of pain and/or focal symptoms with evidence of hemorrhage on MRI, lumbar puncture, or at surgery. In addition, one patient provided such a compelling history involving the single acute onset of pain and hemiparesis, more than ten years prior to his ultimate AVM diagnosis, that a bleeding history was inferred. Three more patients with radiographic evidence of multiple hemorrhagic events also provided similar compelling histories of additional bleeds prior to diagnosis.

\section{Statistical analysis}

A time-dependent Cox proportional-hazards model and SPSS software version 14 were used to analyze the effect of radiosurgery on the incidence of hemorrhage [21-22]. Two sets of timedependent covariates were defined: -1 for the period before radiosurgery and 1 for the period after radiosurgery. The primary endpoint was hemorrhage-free survival. Data on patients prior to radiosurgery was censored at the time of treatment. Patients were censored within the postradiosurgical bleeding risk group when either the AVM obliterated or at last clinical followup. One patient had nearly $95 \%$ of her AVM ablated at five years and underwent embolization to 


\section{Cureus}

obliterate the residual, and was censored at that time. In the overall analysis, patients were also divided into two groups: those initially presenting either with, or without, a prior history of hemorrhage. Next in this analysis, hemorrhage was included as a fixed covariate at the time of initial clinical presentation. To compare the results of these two models, we used the likelihood ratio test to detect interactions between a history of prior hemorrhage and time-dependent covariates.

The reduction in the incidence of hemorrhage in the period after radiosurgery was calculated as $100 \times$ ( 1 - hazard ratio). A two-sided P-value of less than 0.05 was considered to indicate statistical significance. The annual hemorrhage rate was calculated as the number of hemorrhages divided by the aggregate of the all observation periods. The cumulative rate of obliteration was calculated according to the Kaplan-Meier method [23].

\section{Results}

Thirty-three patients with intramedullary spinal cord AVMs were evaluated for inclusion in this study. From within this group, three conservatively followed asymptomatic patients were excluded. Therefore, 30 patients (17 women, 13 men; median age 33 years; range, 9-55) met treatment criteria and underwent CyberKnife radiosurgical ablation of their AVMs (Table 1). Malformations were situated throughout the entire spinal axis; 18 lesions were located in the cervical region of the spinal cord, nine in the thoracic, and three in the conus medullaris. All but one AVM was angiographically consistent with the Type II glomus categorization of spinal cord AVM; the outlier was a Type III thoracic level metameric lesion that included a substantial intramedullary component.

\section{Patient demographics}

Male/female

Location (Cervical/Thoracic/Conus)

Age at radiosurgery (median, range)

Time from diagnosis to radiosurgery (mean, range)

Prior treatment modalities

Embolization 11

Surgical resection

Repeat CyberKnife procedures

\section{$13 / 17$}

$18 / 9 / 3$

33.0 years, (18-55 years)

8.3 years, $(0.2-22$ years $)$

\section{TABLE 1: Patient Demographics}

Presenting symptoms varied with anatomic location in the spinal cord (Table 2). Seventeen patients presented with or had a history of hemorrhage, while pain, vascular steal, or venous congestion caused the presenting symptoms among the remaining cases. Several patients (44\%), especially in the earliest years of this series, had undergone a previous intervention with either embolization (11 patients) and/or surgery (six patients); none underwent prior irradiation. 


\section{Cureus}

\section{Presenting Symptoms}

Hemorrhage

Steal (Transient Neurologic Symptoms)

Pain

Weakness

Sensory Loss

Bladder/Bowel Paresis

Gait Abnormalities

\section{Number (\%)}

$16(57 \%)$

$2(7.4 \%)$

$8(30 \%)$

$16(59 \%)$

$13(48 \%)$

$6(22 \%)$

$11(41 \%)$

\section{TABLE 2: Presenting Symptoms}

The mean time from initial diagnosis to CyberKnife radiosurgery was 8.3 years (range, $0.2-22$ ). Given the rarity of spinal cord AVM, it is not surprising that many of our patients were referred nationally or internationally. No mortality was observed, and no patient was lost to follow-up.

\section{Angiographic response to CyberKnife radiosurgery}

Radiographic response to radiosurgery was ascertained routinely at three years with digital subtraction angiography unless serial MRI suggested earlier obliteration (two patients). The mean clinical and radiographic follow-up after radiosurgery for the entire series was 63 months (median, 54; range, 11 - 145) and 54 months (mean 53; range, 6 - 145), respectively. Twentyseven patients had follow-up greater than 24 months (seven, > seven years; 11, > six years; 11, > five years; 20, >four years; 22, >three years); mean follow up in this group was 66 months (median, 56; range, 24-145). All but four of the 22 patients with greater than three year followup, underwent post-treatment conventional spinal angiography to determine the obliteration status of their AVM. Two such patients refused to undergo angiography; one of whom had a CT angiogram that clearly demonstrated residual, albeit a smaller, AVM, while in second patient flow voids were still visible on MRI. The two remaining patients have angiograms scheduled, but were not completed by the time of this writing. 


\section{Cureus}

34 Patients evaluated

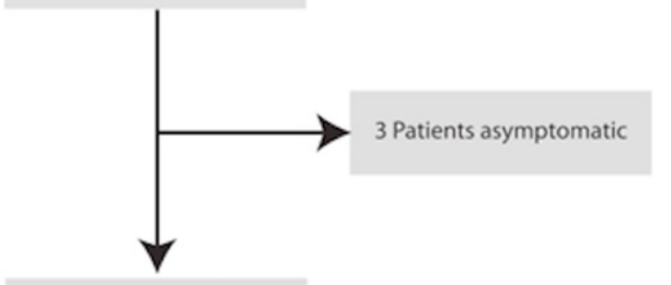

31 Patients underwent radiosurgery

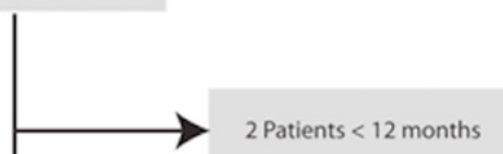

27 Patients with

$>2$ years follow-up

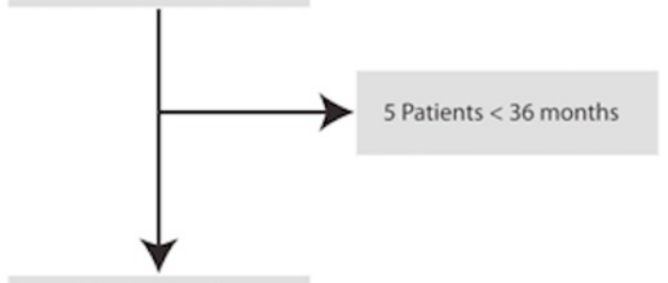

22 Patients with

$>3$ years follow-up

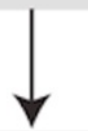

6 SCAVMs obliterated

after first radiosurgery
Figure 1: Flow Diagram of the Study Population.

The observation period was divided into the interval between symptom onset and radiosurgery; between radiosurgery and angiographic obliteration; and from angiographic obliteration and last clinical follow uv.

FIGURE 1: Flow Diagram of the Study Population 


\section{Cureus}

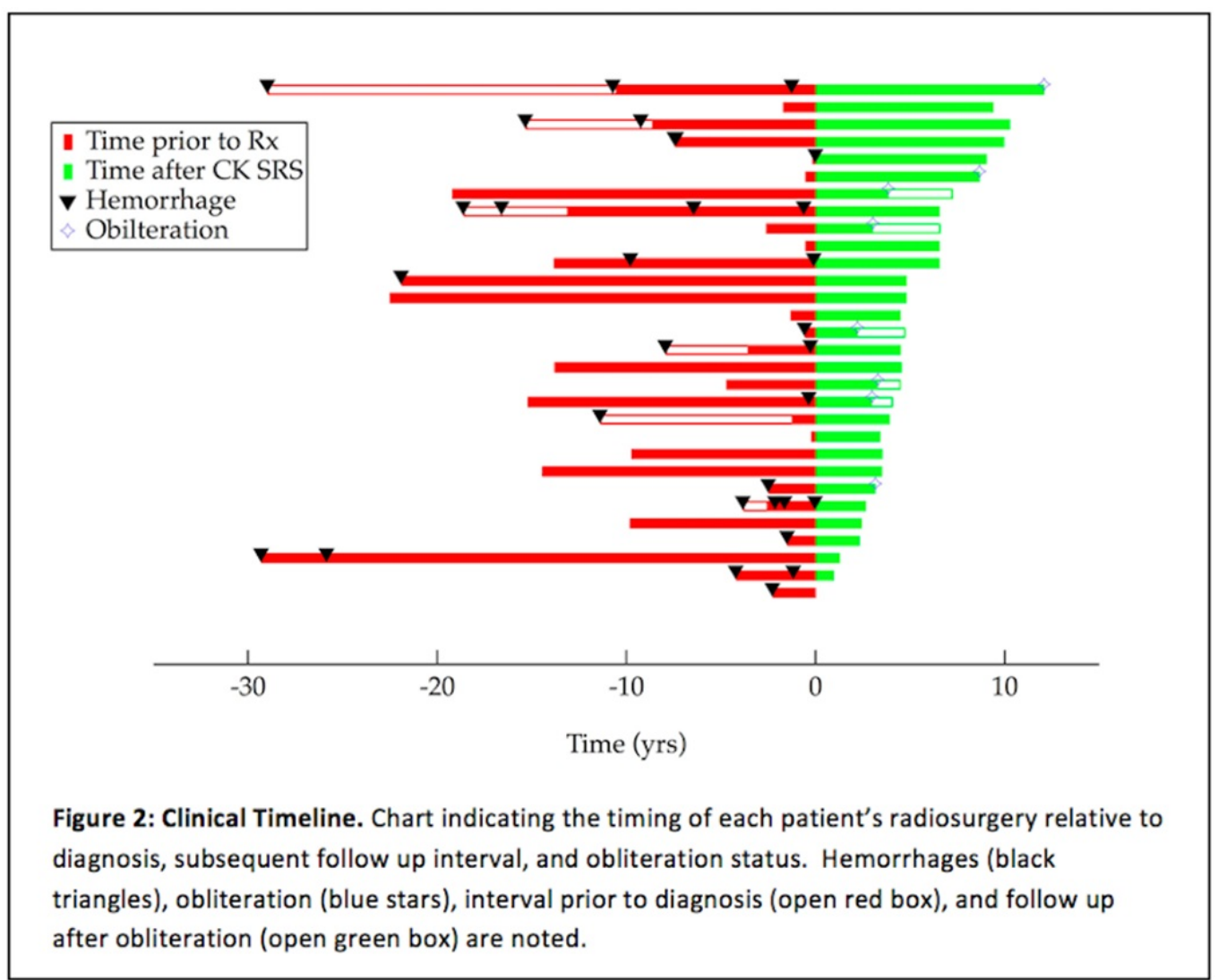

FIGURE 2: Clinical Timeline

Six of 22 patients who were more than three years from a single radiosurgical treatment had complete angiographic obliteration of their AVMs; complete obliteration was defined as no residual nidus or evidence of early venous drainage. The three-year rate of radiosurgical obliteration was therefore $27 \%$. Meanwhile, there was a partial response to radiosurgery in 14 lesions, defined as any reduction in nidal volume greater than $25 \%$, but less than complete obliteration. Within this group, five patients experienced a near complete reduction of nidal volume between $76-95 \%$, five patients had between $51-75 \%$, and in four cases, the decrease in volume was only $25-50 \%$. Any patient in whom the reduction in nidal volume was less than $25 \%$ at three years after radiosurgery was classified as a non-responder (one patient). One patient awaits angiography but had evidence of AVM volume reduction on MRI at two years. These data are summarized in Figure 3. Representative cases are discussed in Figures 4-5. 


\section{Cureus}

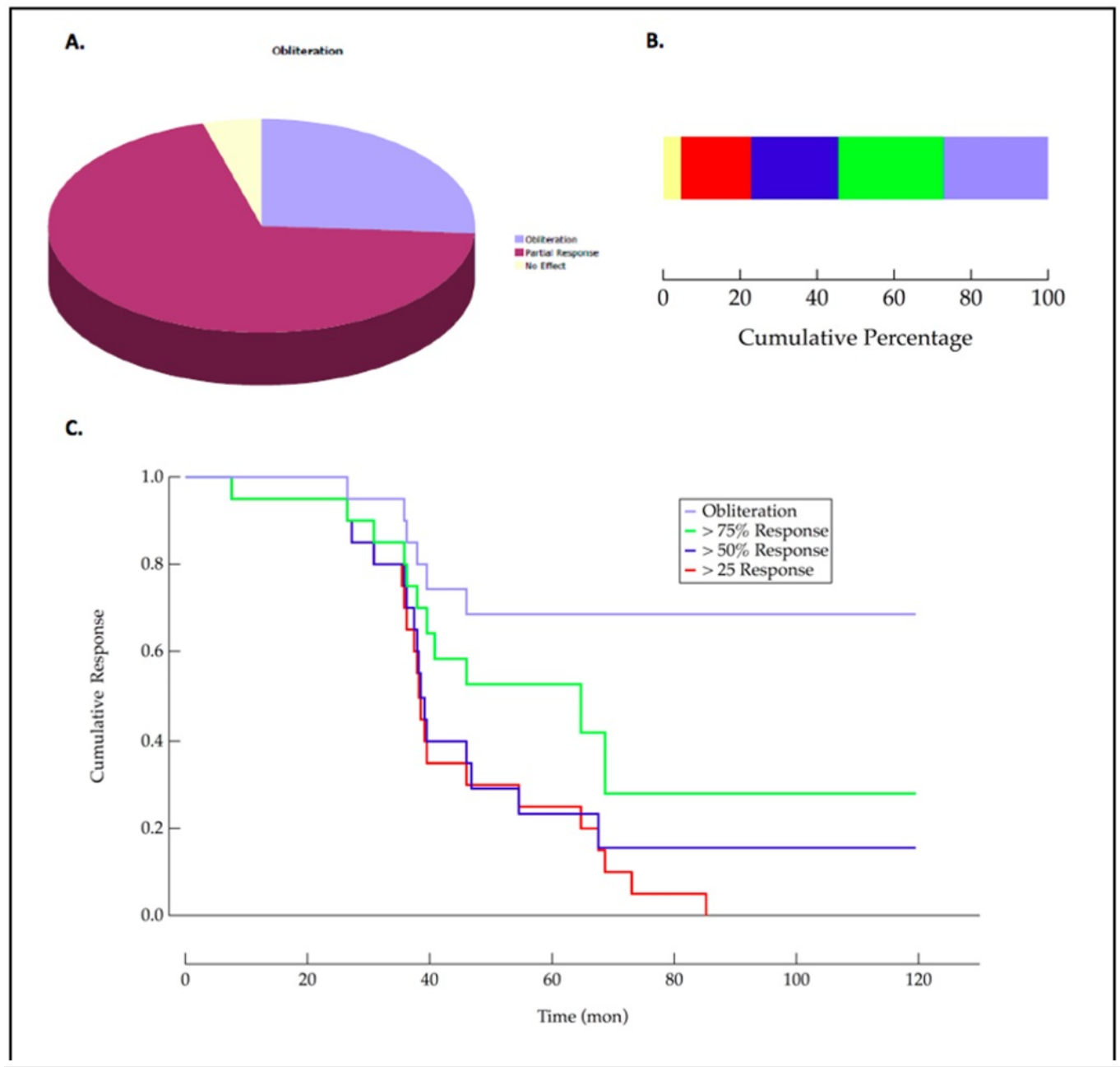

FIGURE 3: Data Visualizations 


\section{Cureus}
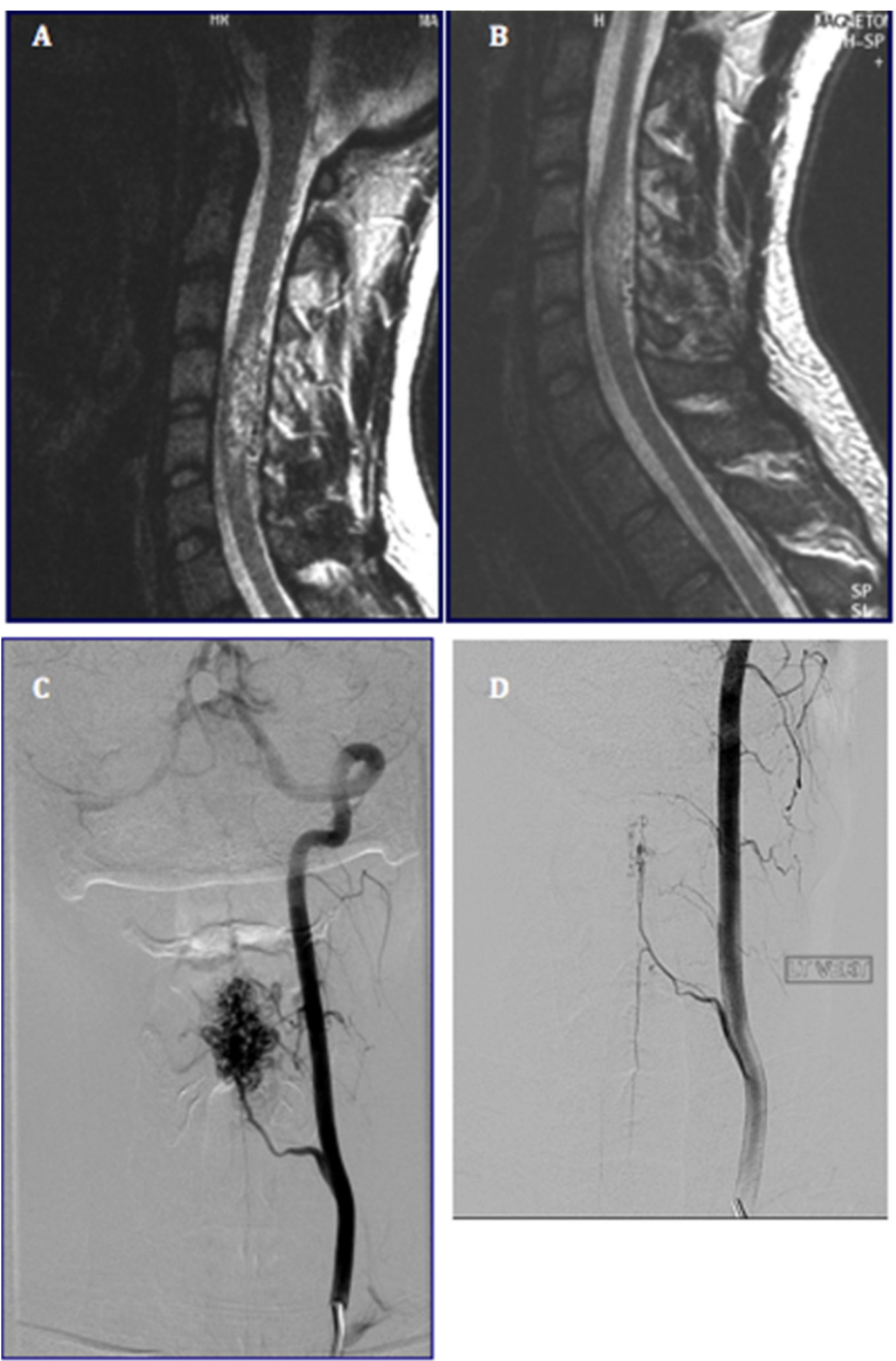

FIGURE 4: Radiographic Response to CyberKnife

\section{Radiosurgery.}

A) preoperative sagittal T2 weighted MRI of C4/5 SCAVM, B) sagittal T2 weighted image 1 year post-SRS, C) preoperative angiogram demonstrating C4/5 SCAVM, D) angiogram demonstrating complete obliteration 3 years post-SRS 


\section{Cureus}

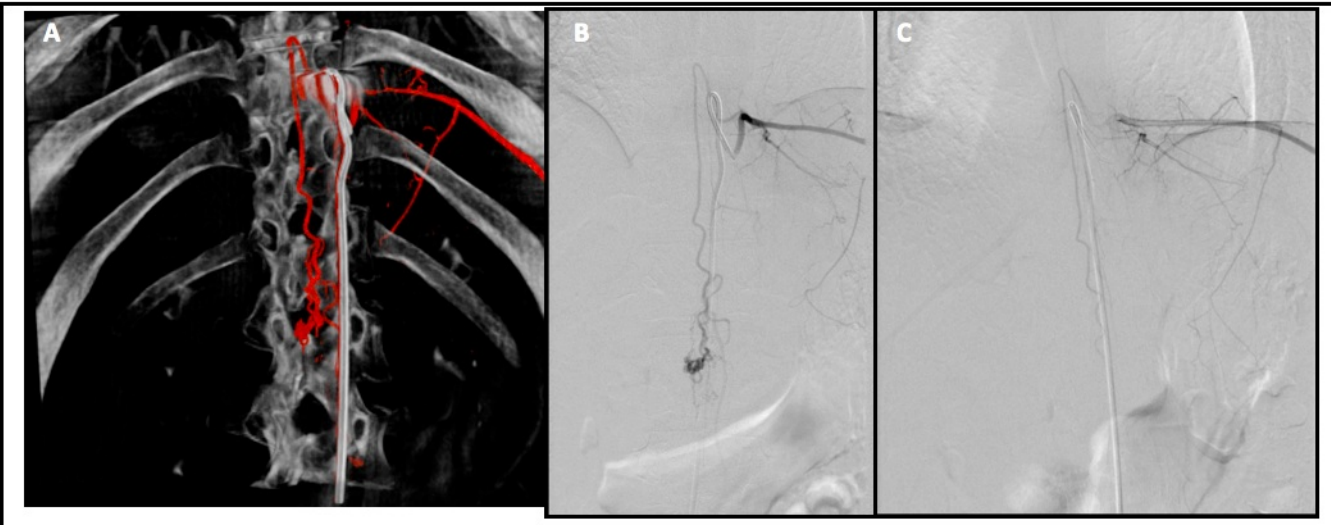

FIGURE 5: Radiographic response of a conus medullaris SCAVM

A) Preoperative 3D reconstruction of conus medullaris AVM, B) AP view of conus SCAVM prior to radiosurgery, C) AP view of feeder vessel injection 3 years post-SRS

No correlation was identified between patient or AVM characteristics and angiographic response to radiosurgery. Despite an expected relationship between AVM volume and angiographic response, none was observed (Figure 6). No correlation was found between spinal cord location and the likelihood of angiographic obliteration (Figure 6). Neither could any relationship be demonstrated between AVM obliteration and patient age, gender, nor prior AVM treatment (not shown).

A

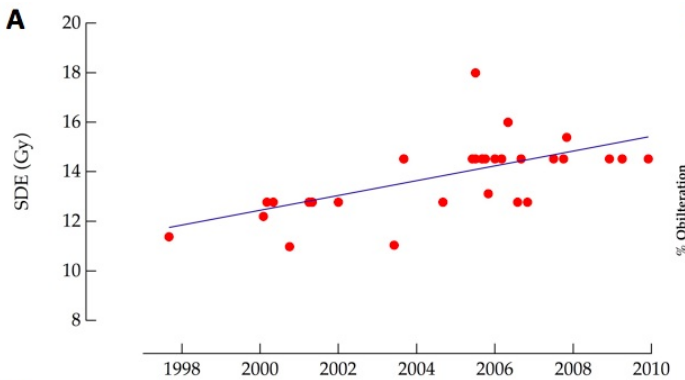

C
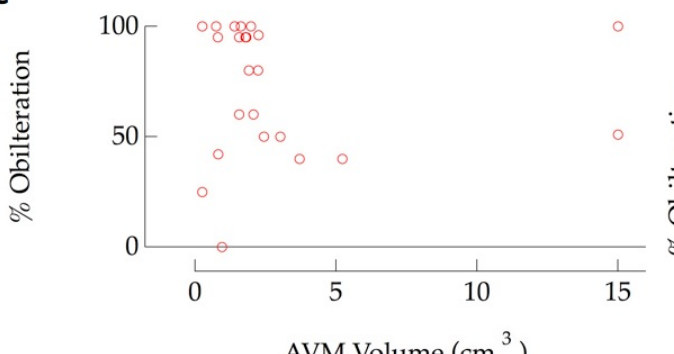
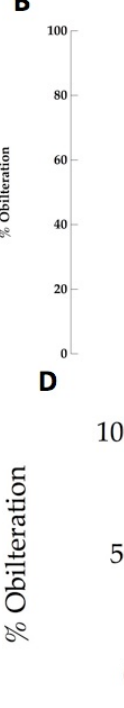

FIGURE 6: Data Visualizations

\section{Radiosurgical dosage and fractionation}

Table 3 summarizes the most relevant radiosurgical parameters used in this series. The target volumes ranged from 0.150 to $15.0 \mathrm{~cm}^{3}$ (mean $2.79 \mathrm{~cm}^{3}$; median $1.70 \mathrm{~cm}^{3}$ ). Treatment plans 


\section{Cureus}

were designed to deliver between 1600 to $2550 \mathrm{cGy}$ to an average $78^{\text {th }}$ percentile isodose line as defined at the margin of the treated AVM. The corresponding maximal intra-lesional dosage ranged from 1190 to $3000 \mathrm{cGy}$ (mean $2500 \mathrm{cGy}$ ). Similar radiosurgical doses were utilized throughout all three regions of the spinal cord.

\begin{tabular}{|c|c|c|c|c|}
\hline \# of Sessions & Total Dose (Gy) & Prescription Isodose & BED (Gy) & Single-dose Equivalent (Gy) \\
\hline 3 & 16.0 & 71 & 58.67 & 9.88 \\
\hline 1 & 16.0 & 71 & 144.00 & 16.00 \\
\hline 1 & 18.0 & 75 & 180.00 & 18.00 \\
\hline 2 & 20.0 & 75 & 120.00 & 14.52 \\
\hline 4 & 21.0 & 70 & 76.13 & 11.38 \\
\hline 2 & 20.0 & 86 & 120.00 & 14.52 \\
\hline 3 & 21.0 & 80 & 94.50 & 12.78 \\
\hline 2 & 20.0 & 80 & 120.0 & 14.52 \\
\hline 2 & 20.0 & 77 & 120.0 & 14.52 \\
\hline 2 & 18.0 & 75 & 99.00 & 13.11 \\
\hline 3 & 20.0 & 80 & 86.67 & 12.20 \\
\hline 2 & 20.0 & 68 & 120.00 & 14.52 \\
\hline 3 & 21.0 & 90 & 94.50 & 12.78 \\
\hline 3 & 20.0 & 70 & 86.67 & 12.20 \\
\hline 3 & 20.0 & 98 & 120.0 & 14.52 \\
\hline 3 & 21.0 & 80 & 94.50 & 12.78 \\
\hline 3 & 20.0 & 74 & 86.67 & 12.20 \\
\hline 3 & 18.0 & 85 & 72.00 & 11.04 \\
\hline 2 & 20.0 & 81 & 120.00 & 14.52 \\
\hline 2 & 15.0 & 85 & 71.25 & 10.98 \\
\hline 2 & 20.0 & 68 & 120.00 & 14.52 \\
\hline 3 & 20.0 & 84 & 86.67 & 12.20 \\
\hline 3 & 21.0 & 86 & 94.50 & 12.78 \\
\hline
\end{tabular}

TABLE 3: Summary of Radiosurgical Parameters 
For small to moderate sized intracranial AVMs, it is generally accepted that the optimal single radiosurgical dose ranges from 18 to $24 \mathrm{~Gy}$ depending on the location of the lesion [24-26]. The choice of dose in this series was an ad hoc decision, drawing from our extensive experience treating AVMs in the brainstem, deep nuclei (38), and extra/intramedullary spinal tumors [16]. Especially early on, a multi-session approach was used to mitigate some of the risk of radiation-induced myelopathy. With experience, we were able to gradually reduce the number of sessions, thereby increasing the prescribed single dose equivalent (Figure $6 a$ ).

Our first patient was treated with $21 \mathrm{~Gy}$ in four sessions (single dose equivalent of about $10 \mathrm{~Gy}$ ) in 1997, after which the dose was gradually increased to $20 \mathrm{~Gy}$ in two sessions, first used in 2002. The single fraction equivalent of this dose is $14.5 \mathrm{~Gy}$, a value that approximates our original dose objective. As we gained greater confidence, two smaller spinal cord AVMs were even treated in recent years using a single session. The most common radiosurgical regimen involved either two (45\%) or three (41\%) sessions. Despite our pre-existing assumption that dose would correlate with the obliteration response, no significant correlation $\left(r^{2}=0.256\right)$ could be demonstrated between BED and the overall angiographic response (Figure $6 b$ ). Nevertheless, it is notable that the six AVMs that were totally obliterated were all treated after 2002, and four of these were treated after 2005 , a time scale which roughly correlates with an increase in radiosurgical dose.

\section{Repeat radiosurgery}

Four patients with incomplete responses at three (two patients), four, and five years from a first radiosurgical treatment were retreated (Figure 7).

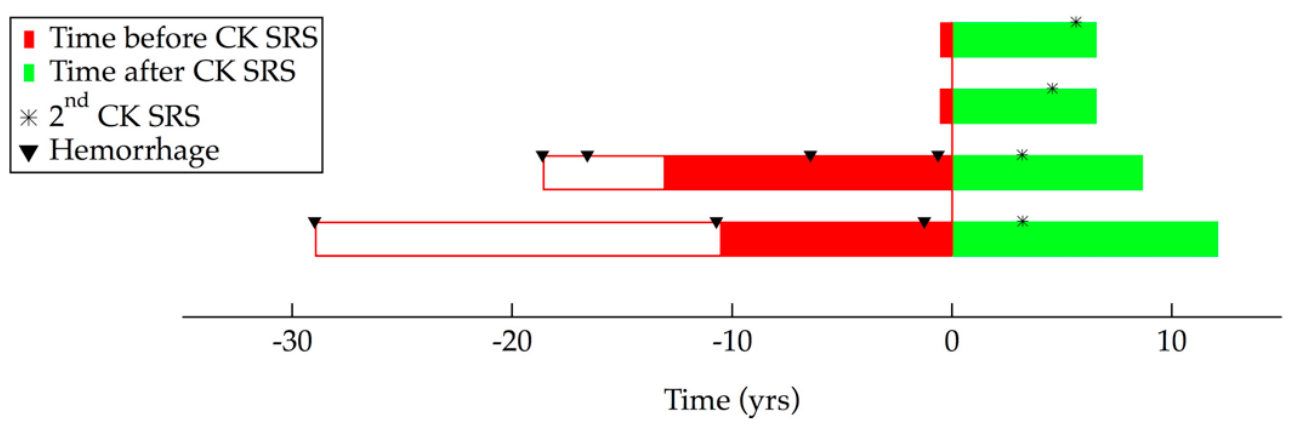

\section{FIGURE 7: Data Visualizations}

Two of the four re-treated AVMs have since gone on to obliterate their AVMs bringing the total number of patients with complete eradication to eight. One patient, the first in this series (1997), who had her C3 AVM treated with a very conservative dose (in hindsight) of 2100 cGy in four sessions. At three years over 50\% of this patient's AVM remained, a size that did not change over the ensuing year. Eight years after being retreated with $1500 \mathrm{cGy}$ in two sessions, follow-up angiography revealed no evidence of residual malformation. Similarly, a second patient, first treated in 2001 for a $1.56 \mathrm{~cm}^{3} \mathrm{C} 3 \mathrm{AVM}$ with $2100 \mathrm{cGy}$ in three sessions, had a 50\% decrease in AVM size on follow-up angiography at 4.5 years. At this juncture, the patient was retreated with $1800 \mathrm{cGy}$ in two sessions. A final angiogram four years later revealed complete occlusion. Two other retreated patients await angiographic follow-up, one of whom has a recent MRI that demonstrates the absence of flow voids and signal characteristics consistent with obliteration. 


\section{Effects of radiosurgery on clinical symptoms}

The small sample size for various symptoms precluded anything except a qualitative assessment. After radiosurgery, most clinical symptoms either remained stable or improved (Figure 8); by 12 months, most patients reported improvement of at least one pre-radiosurgical symptom. Clinical benefits seemed to correlate temporally with a decrease in AVM size and blood flow. At three years of follow-up, a time at which 90\% of AVMs were reduced in size, the most common benefits related to gait ataxia (70\%), myelopathic weakness (50\%), and pain (50\%). The likelihood of improvement in neuropathic pain was significantly higher in this series than previously reported after surgical resection [11, 27]. This difference could reflect the inevitable consequences of separating the posterior columns as is needed to achieve a surgical corridor during microsurgical resection.

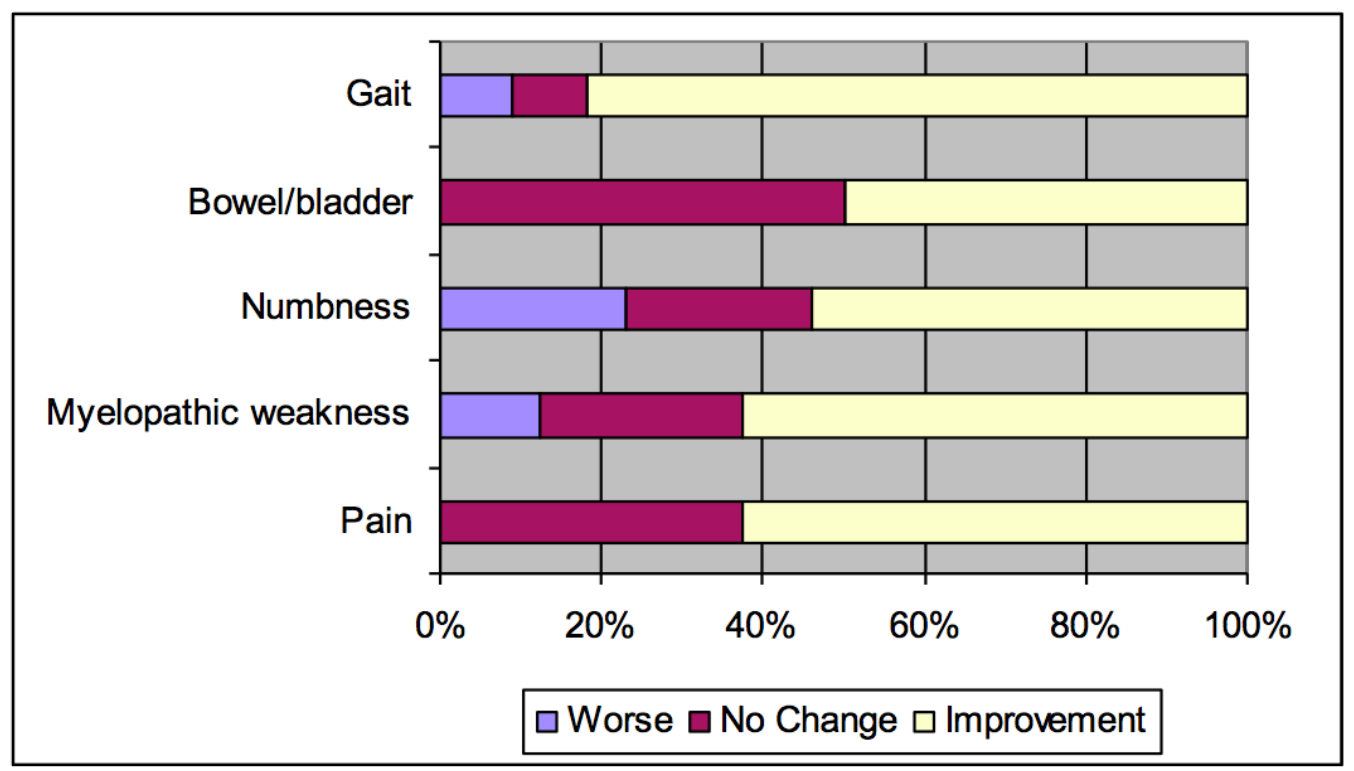

FIGURE 8: Clinical Response to Radiosurgery.

Chart illustrates the effect of radiosurgery on each presenting clinical symptom.

\section{Outcomes in patients with neurological deficits not associated with hemorrhage}

It is has been generally observed that many patients experience improvement in their symptoms within the first year following hemorrhage [3]. Several factors contribute to this phenomenon, including absorption of the hematoma, resolution of edema, increased cord perfusion, and abatement of any inflammatory response. In an effort to understand if there exists any direct clinical benefit from AVM obliteration without a confounding prior history of hemorrhage, outcome was stratified to include a cohort of unruptured spinal cord lesions. Forty-three percent of patients in this study (13 patients) presented with neurological symptoms without evidence of bleeding. In order of decreased frequency, those symptoms were myelopathic weakness (77\%), axial sensory loss (69\%), gait dysfunction (62\%), neuropathic pain (46\%), and bowel/bladder dysfunction (46\%). Symptom improvement, when observed, occurred gradually between six - 24 months. Seventy percent of the non-hemorrhagic patients with weakness and gait disturbance had evidence of benefit following radiosurgery. Pain was also subjectively reported improved in over two-thirds of those with this complaint. The overwhelming majority of patients (80 - 90\%) with dysesthesia and genitourinary dysfunction reported no change or slight improvement in these problems. A single patient in this cohort 
experienced an increase in weakness, while one other complained of a worsened gait. All thirteen patients in this non-hemorrhagic group demonstrated some radiographic response to radiosurgery by two years; on angiography, three patients had complete AVM obliteration, and three had an $80 \%$ or greater reduction in size.

\section{Outcomes in patients with hemorrhagic presentation}

Seven of the seventeen patients with history of AVM bleeding underwent either embolization or microsurgery prior to referral, two of which suffered bleeding events between their last intervention and radiosurgery. Six of seventeen patients received radiosurgery within one year of their last hemorrhage. The mean time between the last bleed and CyberKnife radiosurgery for the entire group was 5.7 years (median, 1.5). Two-year follow-up was available in fifteen of patients in this cohort. When compared to the non-hemorrhagic group, patients with a history of AVM bleed were equally likely to present with weakness (82\%), numbness (76\%), and pain (47\%), but less likely to complain of gait ataxia (35\%), or bowel/bladder dysfunction (18\%). Approximately $50 \%$ of post-hemorrhage patients had no change in their symptoms following radiosurgery, while about one-third reported benefits. Meanwhile, three patients had increased weakness, sensory loss, or neuropathic pain. Among the 12 (out of 17) patients who underwent three-year angiographic follow-up, three had complete AVM obliteration, eight had a partial angiographic response, and one did not respond at all.

\section{Rate of hemorrhage before and after radiosurgery}

Seventeen patients (57\%) presented with a prior history of AVM bleed, and in over 50\%, there were multiple hemorrhages (Figure 2). Three of the seventeen patients presented with other symptoms before suffering a bleed. Despite a history of hemorrhage, intra-nidal aneurysms were observed on angiography in only a minority of cases.

Assuming that the probability of an AVM bleed is linear from the time of first presentation, an annual rate of hemorrhage prior to radiosurgery was estimated by dividing the number of hemorrhages by the sum of the observation period. Among the seventeen patients that bled, 31 pre-radiosurgical hemorrhages were recorded; nine patients had at least two bleeds, three had at least three, and two had four episodes of bleeding. Meanwhile, a total of 305.76 patient years elapsed throughout the entire series (30 cases) between diagnosis and radiosurgery. Given the above values, the retrospectively estimated annual rate of hemorrhage was calculated to be $10 \%$ per patient-year. In contrast, the annual hemorrhage rate prior to treatment in the cohort with a history of hemorrhage was similarly determined to be $17 \%$ per patient-year. When normalized to the initial "index" hemorrhage, the mean duration to re-hemorrhage, which occurred in nine patients, was 5.3 years (median, 3.33).

It is widely assumed that persistence of any AVM nidus on angiography is associated with a risk of future hemorrhage that is little changed from the natural history of an untreated lesion. In other words, only complete obliteration or resection is protective. Despite this general belief, no rebleeding was observed among any of the partially obliterated spinal cord AVMs in the current study, after a mean 5.4 years (median, 4.5) of post-radiosurgical follow-up (Figure 9). Based on this analysis, it appears that CyberKnife radiosurgery reduced the risk of AVM bleeding among this entire series of patients. The decrease in risk was even greater among the 17 patients who presented with a history of hemorrhage. 


\section{Cureus}

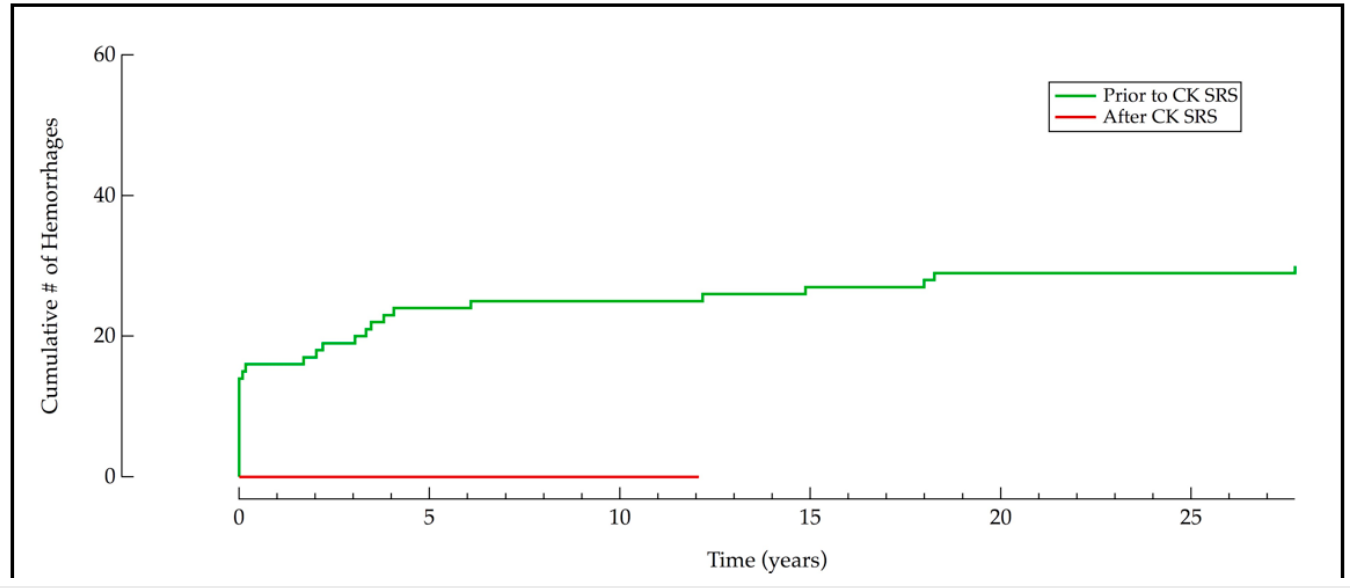

\section{FIGURE 9: Hemorrhage rates before and after CyberKnife radiosurgery.}

No rebleeding was observed in patients treated with SRS

\section{Neurological deterioration post-radiosurgery}

Three patients experienced worsening of their symptoms following CyberKnife radiosurgery. The only direct treatment related complication occurred in a 19 year-old woman with a cervico-thoracic junction AVM, who developed worsening hemiparesis nine months after radiosurgery. In this presumptive case of radiation injury, the total prescribed dose was 2000 cGy administered over two sessions to a lesion volume of $1.57 \mathrm{~cm}^{3}$, using a maximum dose of 2667 cGy. Radiosurgical volume and other treatment parameters in this case were similar to those used for other patients in this series. MRI revealed increased T2 weighted signal within the adjacent spinal cord and other imaging characteristics consistent with radiation-induced myelopathy (Figure 10); meanwhile, angiography demonstrated a greater than $90 \%$ reduction, but not complete obliteration, in AVM nidal volume.
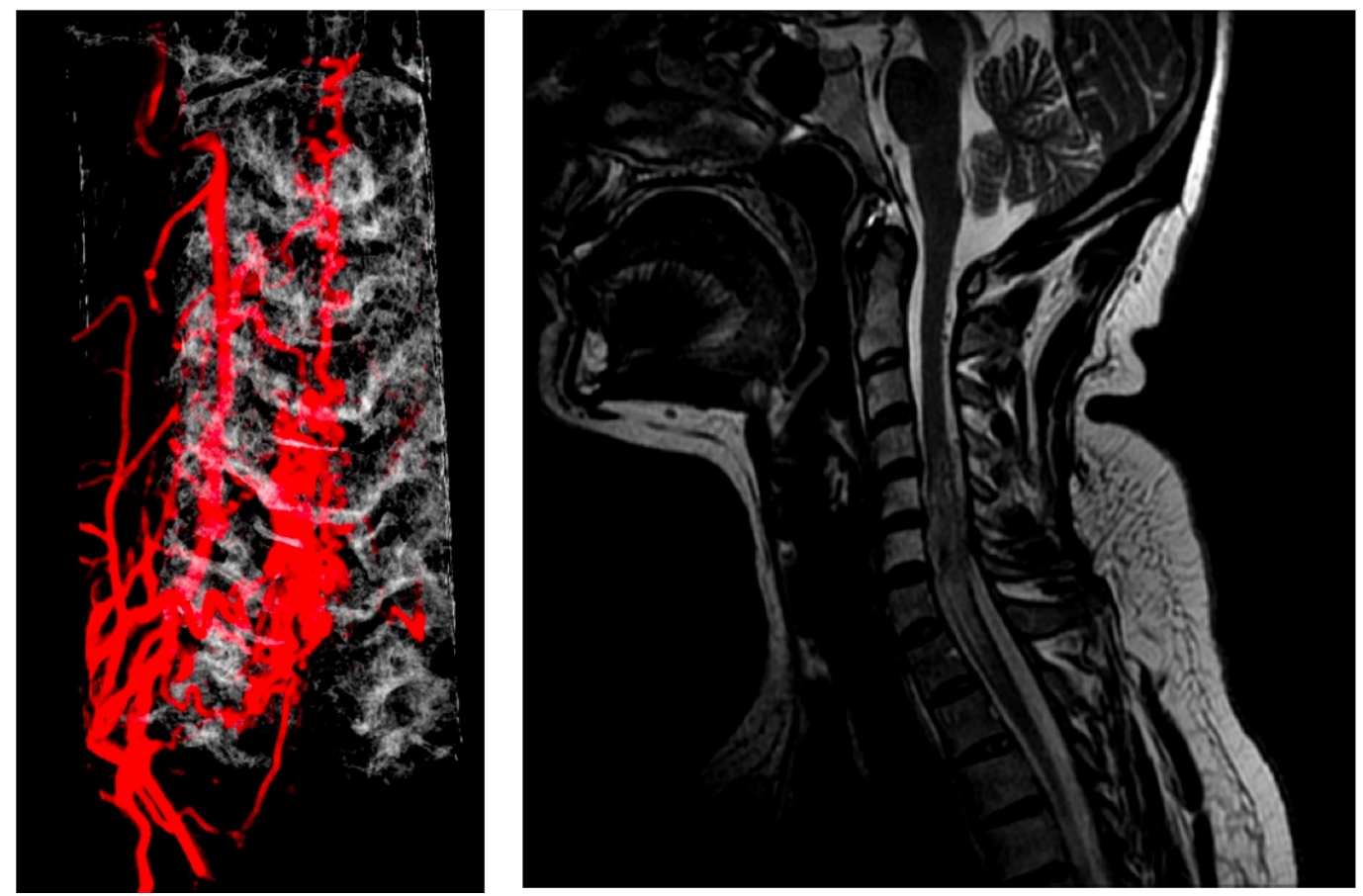


\section{FIGURE 10: Radiation myelopathy.}

A) 3D reconstruction of large lower cervical AVM. B) Radiographic effects of radiation-induced injury to adjacent spinal cord observed 9 months following radiosurgery.

Post-treatment neurological dysfunction was also observed in a 30-year-old man with a T12 glomus AVM who suffered from progressive lower extremity weakness and sensory dysfunction. He underwent CyberKnife SRS in two sessions to a total marginal dose of 2000 cGy. During the second of two sessions (2 x $10 \mathrm{~Gy}$, total $20 \mathrm{~Gy}$ ), he developed an acute flaccid paraparesis. Immediate MRI did not demonstrate hemorrhage or radiation injury. Although the mechanism for such an acute neurological change remains unclear, the patient made a gradual recovery over the ensuing 18 months. At the last follow-up, significant deficits persist but the patient was able to walk without assistance. Angiography at three years confirmed AVM obliteration.

The third patient who suffered neurologic deterioration after radiosurgery is a 35 -year-old woman with a history of multiple hemorrhages from a C5/6 AVM who developed worsening leg weakness, despite prior partial AVM embolization. Three radiosurgical sessions were administered to a total marginal dose of $1800 \mathrm{cGy}$, as defined at the $85 \%$ isodose line. CyberKnife radiosurgery was repeated at three years because of persistent leg symptoms and a 50\% residual AVM nidus. For this second treatment, a marginal prescription dose of 2100 cGy in three sessions, at the $71 \%$ isodose line was utilized. Despite re-treatment, her leg weakness continued to progress, and was eventually accompanied by urinary retention. An MRI done three years after the second course of radiosurgery revealed disappearance of intramedullary flow voids but no explanation for the new neurologic symptoms. Follow-up angiography is scheduled.

\section{Discussion}

Determining the efficacy of an innovative treatment for a rare insidious medical condition with a poorly understood natural history is often a vexing problem. Nevertheless, when there exists the looming possibility of an uncertain but serious injury if the disease is left untreated, physicians are pushed towards some type of intervention. However, in doing so, the underlying natural history is never revealed. Such is the case for stereotactic radiosurgery for spinal cord arteriovenous malformations (SCAVMs), where low event rates (hemorrhages), slow treatment effects, and small numbers of patients, all collectively conspire against the unambiguous demonstration of treatment benefit. Furthermore, despite an uncertain natural history, it is difficult to stay one's surgical instincts in the setting of such a potentially catastrophic neurologic disorder. It was under this scenario that the senior author first began to use CyberKnife radiosurgery to manage spinal cord AVMs in 1996.

The use of CyberKnife radiosurgery to ablate high risk and generally untreatable diffuse intramedullary SCAVMs represented a logical extension of the approach developed for deep intracranial arteriovenous malformations. In this study, which notably utilized the most conservative of radiosurgical doses, we report a three-year angiographic obliteration rate of over $27 \%$, a response rate of over $95 \%$, and a complication rate of less than $10 \%$. These results demonstrate that CyberKnife radiosurgery can be an effective and safe option for many SCAVM.

The overall obliteration rate we observed is significantly below what has been described for intracranial AVMs, which at three to five years has been reported to be as high as 80 to $90 \%$ for malformations less than $3 \mathrm{~cm}$ in diameter [24-25]. However, the radiosurgical doses prescribed in these studies were as much as 12 Gy more than those employed for SCAVMs in the current 
series. It should be noted that the treatment of thousands of brain AVMs was required to arrive at these seemingly optimal present day radiosurgery treatment parameters and corresponding clinical outcomes. Therefore, it would seem likely that still further experience, beyond that which we now report, will be needed to determine the ideal prescription dose for SCAVM. Unfortunately, given the rarity of these lesions, it could take considerable time to acquire the requisite experience.

Despite the modest number of cases in the current study, it is one of the largest series of SCAVM patients undergoing any type of treatment. Among the largest experiences, Spetzler, et al. described the post-microsurgical outcome in 27 patients with intramedullary and 17 conus AVMs; resection was complete in $92 \%$ of cases and 53\% were improved following surgery [10]. Meanwhile, Berenstein and Lasjaunias detail their experience with 44 SCAVMs treated with endovascular embolization. In this series, $53 \%$ of patients had a complete obliteration of their AVM. Although the overall clinical outcome was reported as good or excellent in $77 \%$ of patients, a complication rate of $11 \%$ was encountered [3].

The published experience with any type of radiation treatment for SCAVM is quite limited, consisting of one clinical series [28] and a few single case reports [29-30]. Hida, et al. described a series of 10 patients with SCAVMs who were treated with hypofractionated LINAC stereotactic radiotherapy [16]. The prescription dose varied between $32 \mathrm{~Gy}$ in 18 fractions (1.8 Gy/day) to 40 Gy in 20 fractions (2 Gy/day) in most patients; one patient received $3.75 \mathrm{~Gy} /$ day for eight days (30 Gy), and two patients received $5 \mathrm{~Gy} /$ day for four days (20 Gy). The single fraction equivalent dose to this variety of radiation therapy treatments would range between 10 to $12 \mathrm{~Gy}$, which is substantially less than our initially targeted dosage in the current study. While complete AVM obliteration was not observed after stereotactic radiotherapy, five patients had a partial volume reduction of the nidus on angiography, over a median of 49 months. Given what is known about the dose responsiveness of cerebral AVMs after radiosurgery, it is reasonable to surmise that the same biologic phenomenon explains the marked differences in AVM volume reduction noted between Hida et. al. [16] and the radiosurgical experience we now report.

As argued above, it would seem probable that the extent of shrinkage and the likelihood of spinal cord AVM obliteration are directly related to radiosurgical dose. Despite this seemingly well-founded argument, we were unable to demonstrate such an effect in this series. In large part, this may simply be an artifact that results from the small number of patients that were treated and heterogeneity of the study cohort. In addition, the relatively conservative doses utilized to date may still be situated on "the flat" low dose part of the radiosurgical dose response curve.

Our incomplete knowledge of the radiation tolerance of normal spinal cord was a major influence in our selection of radiation dose. Based on the clinical observations with patients undergoing radiotherapy for head and neck cancers, the dose tolerance of the cervical spinal cord is believed to be $50 \mathrm{~Gy}$ when using conventional fractionation [31]. However, because BED formulations are much less accurate when comparing hypofractionated regimens, it is unclear how to directly translate standard radiotherapy dosing to radiosurgical practice. Since (fortunately) only one patient in this series suffered a complication on a time scale that one might expect from a delayed radiation-induced injury, it is impossible to arrive at firm conclusions about the upper radiation dose limit for normal spinal cord. Nevertheless, the fact that there was only one such apparent injury provides a reasonable rationale for further dose escalation, perhaps especially in patients with the smallest SCAVMs? Despite our still modest understanding of radiation injury thresholds, our current experience, including the small number of SCAVM cases who underwent two separate radiosurgical treatments, clearly demonstrates that conventional teaching underestimates the radiation tolerance of the spinal cord; repair of sub-lethal damage may be a much more potent influence than previously recognized. 
Estimates of the rate of cerebral AVM hemorrhage vary significantly depending on whether the analysis aims to report the cumulative or per annum risk [32-35], and the inclusion of recurrent and previous hemorrhages [7]. Given their relative rarity, confusion among classification systems [10], and the small number of modern clinical series with any natural history data, it is even more difficult to estimate the innate rate of bleeding for SCAVM. Shephard reports a series "intradural AVM" where 39 hemorrhages were observed in 35 patients over a mean 8.5 year interval prior to surgical resection [5]. In this retrospective study, the annual rate of SCAVM hemorrhage among all patients was $13 \%$ per patient year, and $17 \%$ per patient year in the cohort of 22 patients with a history of hemorrhage. Interestingly, this compares similarly to what was observed in the current series; the rate of rebleed over a mean 8.3 years prior to radiosurgery was 10 and $17 \%$ among the two different categories of SCAVM. Notably, both values are considerably higher than the approximate $2.4 \%$ per year annual hemorrhage rate for intracranial AVM [36].

Despite incomplete SCAVM obliteration, we observed a significantly decreased risk of hemorrhage after radiosurgery. Previous studies on intracranial AVMs have reported that the risk of hemorrhage during the latency period decrease [37-38], remains unchanged [36, 39], or even increases $[15,40]$ when compared with the natural course of the disease. Of note, these prior cerebral AVM studies tended to compare the risk of hemorrhage among selected patients who underwent radiosurgery with patients who did not undergo radiosurgery; in contrast, the present series analyzed changes in the rate of hemorrhage pre- and post-radiosurgery. Our findings appear to mirror those reported for intracranial AVMs by Maruyama, et al. [26]. Interestingly, the only other series of SCAVMs treated with radiation also observed a zero rate of hemorrhage among ten patients with non-obliterated lesions followed for at least four years [28].

The traditional belief has been that persistent AVM nidus following radiosurgery portends a hemorrhage risk equivalent to an untreated lesson [41-43]. The fact that no further hemorrhage was observed among treated but non-obliterated AVM in the current series is at odds with this tenet. Although a protective effect of radiosurgery in the absence of obliteration could be artifactual, explained by the small sample population, a relatively brief post-radiosurgery observation interval, or a more benign natural history of SCAVM than previously believed, it could also be a real phenomenon, as best described by Maruyama [26]. Although the sample size is small, the fact that the improvement in clinical symptoms was more prevalent in patients without prior history of hemorrhage, as compared to those with such a bleeding history, may suggest that spinal cord damaged by bleeding tends to recover less well than nervous tissue compromised by mass effect and steal. Hopefully, future studies can examine this finding in greater depth.

\section{Conclusions}

What is the exact role of radiosurgery when multimodal treatment options for SCAVM are available? This question is particularly pertinent to incompletely obliterated lesions as is illustrated by our patient who 5.4 years following $95 \%$ radiosurgical ablation of her thoracic $\mathrm{AVM}$, underwent complete obliteration by means of endovascular embolization. Of note, multiple prior embolization attempts in this patient had been unsuccessful. Moreover, it is unclear whether or not repeat radiosurgery, which was never considered, might have achieved the same definitive outcome in such a case. It is probable that centers which provide surgery, endovascular therapy, and radiosurgery for SCAVMs, will find that similar to brain lesions, a multimodality paradigm is sometimes superior to an approach using any one treatment alone. However, as demonstrated by this paper, a primary radiosurgical approach is often a safe and effective tool for treating most SCAVM. Despite our favorable single institutional experience, a prospective multi-institutional trial, which includes stricter definitions for radiosurgical planning and spinal cord morbidity, is needed to further validate our proposed strategy for 
managing SCAVMs. In particular, still longer term follow-up is required to validate the safety of radiosurgery for intrinsic spinal cord lesions.

\section{Additional Information \\ Disclosures}

Human subjects: Consent was obtained by all participants in this study. The Stanford University Institutional Review Board issued approval N/A. Animal subjects: All authors have confirmed that this study did not involve animal subjects or tissue. Conflicts of interest: In compliance with the ICMJE uniform disclosure form, all authors declare the following: Payment/services info: All authors have declared that no financial support was received from any organization for the submitted work. Financial relationships: John Adler declare(s) an alternate financial activity from Accuray, Inc. Shareholder. Other relationships: All authors have declared that there are no other relationships or activities that could appear to have influenced the submitted work.

\section{References}

1. Rosenblum B, Oldfield E, Doppman L, Di Chiro G: Spinal arteriovenous malformations: a comparison of dural arteriovenous fistulas and intradural AVM's in 81 patients. J Neurosurg. 1987, 67:795-802.

2. Aminoff M, Logue V: Clinical features of spinal vascular malformations . Brain. 1974, 97:197210.

3. Berenstein A, Lasjaunias P, ter Brugge K: Spinal Arteriovenous Malformations; in Berenstein A, Lasjaunias P, ter Brugge KG (eds). Surgical Neuroangiography. 2004, 738-847.

4. Yasargil M, Symon L, Teddy P: Arteriovenous malformations of the spinal cord . Adv Tech Stand Neurosurg. 1984, 11:61-102.

5. Shephard R: Spinal arteriovenous malformations and subarachnoid hemorrhage. $\mathrm{Br} \mathrm{J}$ Neurosurg. 1992, 6:5-12.

6. Malis L: Microsurgery for spinal cord arteriovenous malformations . Clin Neurosurg. 1979, 26:543-555.

7. ApSimon H, Reef H, Phadke R, Popovic E: A population-based study of brain arteriovenous malformation: long-term treatment outcomes. Stroke. 2002, 33:2794-2800.

8. Aminoff M, Logue V: Clinical features of spinal vascular malformations. Brain. 1974, 97:197210.

9. Tobin W, Layton D: The diagnosis and natural history of spinal cord arteriovenous malformations. Mayo Clin Proc. 1976, 51:637-646.

10. Spetzler R, Detwiler P, Riina H, Porter R: Modified classification of spinal cord vascular lesions. J Neurosurg. 2002, 96:145-156.

11. Connolly E, Jr., Zubay G, McCormick P, Stein B: The posterior approach to a series of glomus (Type II) intramedullary spinal cord arteriovenous malformations. Neurosurgery. 1998, 42:774-785.

12. Sinclair J, Chang S, Gibbs I, Adler J, Jr: Multisession CyberKnife radiosurgery for intramedullary spinal cord arteriovenous malformations. Neurosurgery. 2006, 58:1081-1089.

13. Ryu S, Chang S, Kim D, Murphy M, Le Q, Martin D, Adler J, Jr.: Image-guided hypofractionated stereotactic radiosurgery to spinal lesions. Neurosurgery. 2001, 49:838-846.

14. Prestigiacomo C, Niimi Y, Setton A, Berenstein A: Three-dimensional rotational spinal angiography in the evaluation and treatment of vascular malformations. AJNR Am J Neuroradiol. 2003, 24:1429-1435.

15. Steinberg G, Fabrikant J, Marks M, Levy R, Frankel K, Phillips M, Shuer L, Silverberg G: Stereotactic heavy-charged-particle Bragg-peak radiation for intracranial arteriovenous malformations. N Engl J Med. 1990, 323:96-101.

16. Dodd R, Ryu M, Kamnerdsupaphon P, Gibbs I, Chang S, Adler J Jr: CyberKnife radiosurgery for benign intradural extramedullary spinal tumors. Neurosurgery. 2006, 58:674-685.

17. Poen J, Golby A, Forster K, Martin D, Chinn D, Hancock S, Adler J Jr: Fractionated stereotactic radiosurgery and preservation of hearing in patients with vestibular schwannoma: a 
preliminary report. Neurosurgery. 1999, 45:1299-1305.

18. Hall E, Brenner D: The radiobiology of radiosurgery: rationale for different treatment regimens for AVMs and malignancies. Int J Radiat Oncol Biol Phys. 1993, 25:381-385.

19. Flickinger J, Kondziolka D, Maitz A, Lunsford L: An analysis of the dose-response for arteriovenous malformation radiosurgery and other factors affecting obliteration. Radiother Oncol. 2002, 63:347-354.

20. Marks M, Lane B, Steinberg G, Chang P: Hemorrhage in intracerebral arteriovenous malformations: angiographic determinants. Radiology. 1990, 176:807-813.

21. Crowley J, Hu M: Covariance analysis of heart-transplant survival data . J Am Stat Assoc. 1977, 72:27-36.

22. Cox D: Regression models and life-tables. J R Stat Soc. 1972, 34:187-220.

23. Kaplan E, Meier P: Nonparametric estimation from incomplete observations. J Am Stat Assoc. 1958, 53:457-481.

24. Pollock B, Flickinger J, Lunsford L, Maitz A, Kondziolka D: Factors associated with successful arteriovenous malformation radiosurgery. 1239-1244. 1998, 42:1239-1244.

25. Pollock B, Gorman D, Brown P: Radiosurgery for arteriovenous malformations of the basal ganglia, thalamus, and brainstem. J Neurosurg. 2004, 100:210-214.

26. Maruyama K, Kawahara N, Shin M, Tago M, Kishimoto J, Kurita H, Kawamoto S, Morita A, Kirino T: The risk of hemorrhage after radiosurgery for cerebral arteriovenous malformations . N Engl J Med. 2005, 352:146-153.

27. Kalkman C, Drummond J, Hoi S: Severe Sensory Deficits with Preserved Motor Function After Removal of a Spinal Arteriovenous Malformation: Correlation with Simultaneously Recorded Somatosensory and Motor Evoked Potentials. Anesth Analg. 1994, 78:165-168.

28. Hida K, Shirato H, Isu T, Seki T, Onimaru R, Aoyama H, Ushikoshi S, Miyasaka K, Iwasaki Y: Focal fractionated radiotherapy for intramedullary spinal arteriovenous malformations: 10year experience. J Neurosurg. 2003, 99:34-38.

29. Kishi K, Shirai S, Sonomura T, Sato M: Selective conformal radiotherapy for arteriovenous malformation involving the spinal cord. Br J Radiol. 2005, 78:252-254.

30. Wolkov H, Bagshaw M: Conventional radiation therapy in the management of arteriovenous malformations of the central nervous system. Int J Radiat Oncol Biol Phys. 1988, 15:14611464.

31. Emami B, Lyman J, Brown A, Coia L, Goitein M, Munzenrider J, Shank B, Solin L, Wesson M: Tolerance of normal tissue to therapeutic irradiation. Int J Radiat Oncol Biol Phys. 1991, 21:109-122.

32. Brown R, Jr., Wiebers D, Torner J, O'Fallon W: Frequency of intracranial hemorrhage as a presenting symptom and subtype analysis: a population-based study of intracranial vascular malformations in Olmsted Country, Minnesota. J Neurosurg. 1996, 85:29-32.

33. Brown R, Wiebers D, Torner J, O'Fallon W: Incidence and prevalence of intracranial vascular malformations in Olmsted County, Minnesota, 1965 to 1992. Neurology. 1996, 46:949-952.

34. Yamane F, Takeshita M, Izawa M, Kagawa M, Sato K, Takakura K: Natural history of arteriovenous malformations: analysis of non-radically treated patients. J Clin Neurosci. 1998, 5:26-29.

35. Karlsson B, Lindquist C, Johansson A, Steiner L: Annual risk for the first hemorrhage from untreated cerebral arteriovenous malformations. Minim Invasive Neurosurg. 1997, 40:40-46.

36. Pollock B, Flickinger J, Lunsford L, Bissonette D, Kondziolka D: Hemorrhage risk after stereotactic radiosurgery of cerebral arteriovenous malformations. Neurosurgery. 1996, 38:652-659.

37. Karlsson B, Lindquist C, Steiner L: Effect of Gamma Knife surgery on the risk of rupture prior to AVM obliteration. Minim Invasive Neurosurg. 1996, 39:21-27.

38. Levy R, Fabrikant J, Frankel K, Phillips M, Lyman J: Stereotactic heavy-charged-particle Bragg peak radiosurgery for the treatment of intracranial arteriovenous malformations in childhood and adolescence. Neurosurgery. 1989, 24:841-852.

39. Friedman W, Blatt D, Bova F, Buatti J, Mendenhall W, Kubilis P: The risk of hemorrhage after radiosurgery for arteriovenous malformations. J Neurosurg. 1996, 84:912-919.

40. Fabrikant J, Levy R, Steinberg G, Phillips M, Frankel K, Silverberg G: Stereotactic chargedparticle radiosurgery: clinical results of treatment of 1200 patients with intracranial arteriovenous malformations and pituitary disorders. Clin Neurosurg. 1992, 38:472-492.

41. Kjellberg R, Davis K, Lyons S, Butler W, Adams R: Bragg peak proton beam therapy for 
Cureus

arteriovenous malformation of the brain. Clin Neurosurg . 1983, 31:248-290.

42. Steiner L, Lindquist C, Adler J Jr., Torner J, Alves W, Steiner M: Clinical outcome of radiosurgery for cerebral arteriovenous malformations. J Neurosurg. 1992, 77:1-8.

43. Karlsson B, Lax I, Soderman M: Risk for hemorrhage during the 2-year latency period following gamma knife radiosurgery for arteriovenous malformations. Int J Radiat Oncol Biol Phys. 2001, 49:1045-1051. 